\title{
INTEGRALS INVOLVING A MODIFIED BESSEL FUNCTION OF THE SECOND KIND AND AN $E$-FUNCTION
}

\author{
by T. M. MACROBERT
}

(Received 6th April, 1954)

$\S 1$. Introductory. The first formula to be proved is

$$
\int_{0}^{\infty} t^{k-1} K_{n}(t) E\left(p ; \alpha_{r}: q ; \rho_{s}: z t\right) d t=2^{\Sigma \alpha_{r}-\Sigma \rho_{s}}(\sqrt{ } \pi)^{5-p+q}
$$

$\left[\begin{array}{c}\frac{2^{k-p+q-2}}{\sin \left(\frac{k+n}{2} \pi\right) \sin \left(\frac{k-n}{2} \pi\right)} E\left(\begin{array}{c}\frac{\alpha_{1}}{2}, \frac{\alpha_{1}+1}{2}, \ldots, \frac{\alpha_{p}+1}{2}: e^{ \pm i \pi} 4^{q-p+2} z^{2} \\ \left.\frac{1}{2}, 1-\frac{k+n}{2}, 1-\frac{k-n}{2}, \frac{\rho_{1}}{2}, \ldots, \frac{\rho_{q}+1}{2}\right)\end{array}\right) \\ -\frac{2^{k-4}}{\cos \left(\frac{k+n}{2} \pi\right) \cos \left(\frac{k-n}{2} \pi\right)} z^{-1} E\left(\begin{array}{c}\frac{\alpha_{1}+1}{2}, \frac{\alpha_{1}+2}{2}, \ldots, \frac{\alpha_{p}+2}{2}: e^{ \pm i \pi} 4^{q-p+2} z^{2} \\ \frac{3}{2}, \frac{3-k-n}{2}, \frac{3-k+n}{2}, \frac{\rho_{1}+1}{2}, \ldots, \frac{\rho_{q}+2}{2}\end{array}\right) \\ +\sum_{n,-n} \frac{2^{-k-2 n-1+(k+n-1)(p-a)}}{\sin n \pi \sin (k+n) \pi} z^{-k-n} \\ \times E\left(\begin{array}{c}\frac{\alpha_{1}+k+n}{2}, \frac{\alpha_{1}+k+n+1}{2}, \ldots, \frac{\alpha_{p}+k+n+1}{2}: e^{ \pm i \pi} 4^{\alpha-p+2} z^{2} \\ n+1, \frac{k+n+1}{2}, \frac{k+n+2}{2}, \frac{\rho_{1}+k+n}{2}, \ldots, \frac{\rho_{\alpha}+k+n+1}{2}\end{array}\right)\end{array}\right]$

where $p \geqq q+1,|\operatorname{amp} z|<\pi, R\left(k \pm n+\alpha_{r}\right)>0, r=1,2, \ldots, p$. For other values of $p$ and $q$ the result is valid if the integral is convergent. A second formula is given in $\S 3$.

The following formulae are required in the proof :

$$
\begin{aligned}
& \int_{0}^{\infty} t^{l-1} K_{n}(t) K_{m}(z / t) d t \\
& =\sum_{m,-m} 2^{l+2 m-3} \Gamma(m) \Gamma\left(\frac{l+m+n}{2}\right) \Gamma\left(\frac{l+m-n}{2}\right) z^{-m} \\
& \times F\left(; 1-m, 1-\frac{l+m+n}{2}, 1-\frac{l+m-n}{2} ; \frac{z^{2}}{16}\right) \\
& +\sum_{n,-n} 2^{-l-2 n-3} \Gamma(-n) \Gamma\left(\frac{-l+m-n}{2}\right) \Gamma\left(\frac{-l-m-n}{2}\right) z^{l+n} \\
& \times F\left(; 1+n, 1+\frac{l-m+n}{2}, 1+\frac{l+m+n}{2} ; \frac{z^{2}}{16}\right) \text {, }
\end{aligned}
$$


where $R(z)>0$, (1) ;

$$
\begin{aligned}
& K_{1}(z)=\sqrt{ }\left(\frac{\pi}{2 z}\right) e^{-z}=\sqrt{ }\left(\frac{\pi}{2 z}\right) E\left(:: \frac{1}{z}\right) \\
& \int_{0}^{\infty} e^{-\lambda} \lambda^{\alpha-1} E\left(p ; \alpha_{r}: q ; \rho_{s}: z / \lambda^{2}\right) d \lambda=2^{\alpha-1} \pi^{-1} E\left\{\alpha_{1}, \ldots, \alpha_{p}, \alpha / 2,(\alpha+1) / 2: q ; \rho_{s}: \frac{1}{4} z\right),
\end{aligned}
$$

where $R(\alpha)>0,|\operatorname{amp} z|<\pi,(2)$;

$\frac{1}{2 \pi i} \int e^{\zeta \zeta-\rho} E\left(p ; \alpha_{r}: q ; \rho_{s}: z \zeta^{2}\right) d \zeta=2^{1-\rho^{\frac{1}{b}}} E\left(p ; \alpha_{r}: \rho_{1}, \ldots, \rho_{a}, \rho / 2,(\rho+1) / 2: 4 z\right)$,

where the contour starts from $-\infty$ on the $\xi$-axis, passes round the origin in the positive direction, and ends at $-\infty$ on the $\xi$-axis, the initial value of amp $\zeta$ being $-\pi,(3)$.

$\S 2$. Proof of the Formula. In (2) put $m=\frac{1}{2}$, and apply (3), replacing $l$ by $k-\frac{1}{2}$ and $z$ by $1 / z$; then, on multiplying by $\sqrt{ }\{2 /(\pi z)\}$, it is found that, if $R(z)>0$,

$$
\begin{aligned}
& \int_{0}^{1} t^{k-1} K_{n}(t) E(:: z t) d t \\
& =\frac{2^{k-2} \pi^{5 / 2}}{\sin \left(\frac{k+n}{2} \pi\right) \sin \left(\frac{k-n}{2} \pi\right)} E\left(: \frac{1}{2}, 1-\frac{k+n}{2}, 1-\frac{k-n}{2}: e^{ \pm i \pi} 16 z^{2}\right) \\
& -\frac{2^{k-4} \pi^{5 / 2}}{\cos \left(\frac{k+n}{2} \pi\right) \cos \left(\frac{k-n}{2} \pi\right)} z^{-1} E\left(: \frac{3}{2}, \frac{3-k-n}{2}, \frac{3-k+n}{2}: e^{ \pm i \pi} 16 z^{2}\right) \\
& +\sum_{n,-n} \frac{2^{-k-2 n-1} \pi^{5 / 2}}{\sin n \pi \sin (k+n) \pi} z^{-k-n} E\left(: n+1, \frac{k+n+1}{2}, \frac{k+n+2}{2}: e^{ \pm i \pi} 16 z^{2}\right) .
\end{aligned}
$$

On generalising the $E$-function on the left in the usual way, and applying formulae (4) and (5) to the $E$-functions on the right, formula (1) is obtained. When it is necessary to avoid values of $z \zeta$ such that $R(z \zeta) \ngtr 0$, the contour in (5) may be replaced by a line to the right of the origin parallel to the $\eta$-axis, $z$ being taken real and positive. This restriction on $z$ and any restrictions on the $\rho$ 's required for convergence may subsequently be removed by analytical continuation.

§ 3. A Second Integral. The formula to be proved is

$$
\begin{aligned}
& \int_{0}^{\infty} e^{-\lambda} \lambda^{k-1} K_{n}(\lambda) E\left(p ; \alpha_{r}: q ; \rho_{s}: z \lambda\right) d \lambda \\
& =\frac{\pi^{3 / 2} 2^{-k} \cos k \pi}{\sin (k+n) \pi \sin (k-n) \pi} E\left(\begin{array}{l}
\frac{1}{2}-k, \alpha_{1}, \ldots, \alpha_{p}: \frac{1}{2} e^{ \pm i \pi} z \\
1-k+n, 1-k-n, \rho_{1}, \ldots, \rho_{q}
\end{array}\right) \\
& +\sum_{n,-n} \frac{\pi^{3 / 2} 2^{n-1} z^{-k-n}}{\sin (k+n) \pi \sin n \pi} E\left(\begin{array}{l}
\frac{1}{2}+n, \alpha_{1}+k+n, \ldots, \alpha_{p}+k+n: \frac{1}{2} e^{ \pm i \pi} z \\
1+2 n, 1+k+n, \rho_{1}+k+n, \ldots, \rho_{q}+k+n
\end{array}\right)
\end{aligned}
$$

where $p \geqq q+1, R\left(k \pm n+\alpha_{\tau}\right)>0, r=1,2, \ldots, p, \mid$ amp $z \mid<\pi$. For other values of $p$ and $q$ the result is valid if the integral is convergent. 
This result can be derived from Ragab's formula (4)

$$
\begin{aligned}
& \int_{0}^{\infty} e^{-\lambda} \lambda^{k-1} K_{n}(\lambda) K_{m}(z / \lambda) d \lambda \\
& =\sum_{m,-m} \frac{\pi^{3} \cos (k+m) \pi 2^{-3 / 2}(2 / z)^{m}}{\sin (k+m+n) \pi \sin (k+m-n) \pi \sin m \pi} \\
& \times E\left(\begin{array}{l}
\frac{1}{4}-\frac{1}{2} k-\frac{1}{2} m, \frac{3}{4}-\frac{1}{2} k-\frac{1}{2} m: e^{ \pm i \pi} 4 / z^{2} \\
1-m, 1-\frac{1}{2} k-\frac{1}{2} m-\frac{1}{2} n, 1-\frac{1}{2} k-\frac{1}{2} m+\frac{1}{2} n, \frac{1}{2}-\frac{1}{2} k-\frac{1}{2} m-\frac{1}{2} n, \frac{1}{2}-\frac{1}{2} k-\frac{1}{2} m+\frac{1}{2} n
\end{array}\right) \\
& +\sum_{n,-n}\left\{\begin{array}{c}
-\frac{\pi^{32-7 / 2}(z / 2)^{k+n}}{\sin \left(\frac{k-m+n}{2} \pi\right) \sin \left(\frac{k+m+n}{2} \pi\right) \sin n \pi} \\
\times E\left(\begin{array}{c}
\frac{1}{4}+\frac{1}{2} n, \frac{3}{4}+\frac{1}{2} n: e^{ \pm i \pi} 4 / z^{2} \\
1+\frac{1}{2} k+\frac{1}{2} m+\frac{1}{2} n, 1+\frac{1}{2} k-\frac{1}{2} m+\frac{1}{2} n, \frac{1}{2}, \frac{1}{2}+n, 1+n
\end{array}\right) \\
+\frac{\pi^{3} 2^{-7 / 2}(z / 2)^{k+n+1}}{\cos \left(\frac{k-m+n}{2}\right) \cos \left(\frac{k+m+n}{2} \pi\right) \sin n \pi} \\
\times E\left(\frac{\frac{3}{4}+\frac{1}{2} n, \frac{5}{4}+\frac{1}{2} n: e^{ \pm i \pi} 4 / z^{2}}{\frac{3}{2}+\frac{1}{2} k+\frac{1}{2} m+\frac{1}{2} n, \frac{3}{2}+\frac{1}{2} k-\frac{1}{2} m+\frac{1}{2} n, \frac{3}{2}, 1+n, \frac{3}{2}+n}\right)
\end{array}\right\}
\end{aligned}
$$

where $R(z)>0$.

The formula

$E\left(p ; \alpha_{r}: q ; \rho_{s}: e^{ \pm i \pi} z\right)=2^{\Sigma \alpha_{r}-\Sigma \rho_{s}}(\sqrt{ } \pi)^{q-p+1}$

$$
\times\left\{\begin{array}{l}
2^{q-p} E\left(\frac{\alpha_{1}}{2}, \frac{\alpha_{1}+1}{2}, \ldots, \frac{\alpha_{p}+1}{2}: \frac{1}{2}, \frac{\rho_{1}}{2}, \frac{\rho_{1}+1}{2}, \ldots, \frac{\rho_{q}+1}{2}: e^{ \pm i \pi} 4^{q-p+1} z^{2}\right) \\
+\frac{1}{2 z} E\left(\frac{\alpha_{1}+1}{2}, \frac{\alpha_{1}^{\prime}+2}{2}, \ldots, \frac{\alpha_{p}+2}{2}: \frac{3}{2}, \frac{\rho_{1}+1}{2}, \frac{\rho_{1}+2}{2}, \ldots, \frac{\rho_{q}+2}{2}: e^{ \pm i \pi} 4^{q-p+1} z^{2}\right)
\end{array}\right\}
$$

will be required. It can be derived by generalising the formula

$$
e^{1 / z}=E(::-z)=\Gamma\left(\frac{1}{2}\right) E\left(: \frac{1}{2}:-4 z^{2}\right)+\Gamma\left(\frac{3}{2}\right) \frac{1}{z} E\left(: \frac{3}{2}:-4 z^{2}\right) .
$$

Now in (7) replace $z$ by $1 / z$, put $m=\frac{1}{2}$, so that, from (3), $K_{\frac{1}{d}}\{1 /(\lambda z)\}=\sqrt{ }\left(\frac{1}{2} \pi z \lambda\right) E(:: z \lambda)$, replace $k$ by $k-\frac{1}{2}$ and multiply by $\sqrt{ }\{2 /(\pi z)\}$ : then

$$
\begin{aligned}
& \int_{0}^{\infty} e^{-\lambda} \lambda^{k-1} K_{n}(\lambda) E(:: z \lambda) d \lambda=\frac{\pi^{5 / 2} \cos k \pi 2^{-1}}{\sin (k+n) \pi \sin (k-n) \pi} \\
& \quad \times\left\{E\left(\begin{array}{cc}
\frac{1}{4}-\frac{1}{2} k, \frac{3}{4}-\frac{1}{2} k & : e^{ \pm i \pi} 4 z^{2} \\
\frac{1}{2}, 1-\frac{1}{2} k-\frac{1}{2} n, 1-\frac{1}{2} k+\frac{1}{2} n, \frac{1}{2}-\frac{1}{2} k-\frac{1}{2} n, \frac{1}{2}-\frac{1}{2} k+\frac{1}{2} n
\end{array}\right)\right. \\
& \left.\quad+\frac{1}{2 z} E\left(\begin{array}{c}
\frac{3}{4}-\frac{1}{2} k, \frac{5}{4}-\frac{1}{2} k: e^{ \pm i n} 4 z^{2} \\
\left.\left.\frac{3}{2}, \frac{3}{2}-\frac{1}{2} k-\frac{1}{2} n, \frac{3}{2}-\frac{1}{2} k+\frac{1}{2} n, 1-\frac{1}{2} k-\frac{1}{2} n, 1-\frac{1}{2} k+\frac{1}{2} n\right)\right\}
\end{array}\right)\right\} \\
& +\sum_{n,-n} \frac{\pi^{5 / 2} 2^{-3 / 2}(2 z)^{-k-n}}{\sin (k+n) \pi \sin n \pi}\left\{E\left(\begin{array}{c}
\frac{1}{4}+\frac{1}{2} n, \frac{3}{4}+\frac{1}{2} n: e^{ \pm i \pi} 4 z^{2} \\
\frac{1}{2}+\frac{1}{2} k+\frac{1}{2} n, 1+\frac{1}{2} k+\frac{1}{2} n, \frac{1}{2}, \frac{1}{2}+n, 1+n
\end{array}\right)\right. \\
& \left.\quad+\frac{1}{2 z} E\left(\begin{array}{l}
\frac{3}{4}+\frac{1}{2} n, \frac{5}{4}+\frac{1}{2} n: e^{ \pm i \pi} 4 z^{2} \\
1+\frac{1}{2} k+\frac{1}{2} n, \frac{3}{2}+\frac{1}{2} k+\frac{1}{2} n, \frac{3}{2}, 1+n, \frac{3}{2}+n
\end{array}\right)\right\},
\end{aligned}
$$

where $R(z)>0$. 
Here apply (8), and formula (6) with $p=q=0$ is obtained ; formula (6) can then be derived in the usual way.

In particular, on putting $p=2, q=0, \alpha_{1}=\frac{1}{2}+m, \alpha_{2}=\frac{1}{2}-m$, replacing $z$ by $2 z$ and $k$ by $k-\frac{1}{2}$, and applying the formula

$$
\cos m \pi E\left(\frac{1}{2}+m, \frac{1}{2}-m:: 2 z\right)=\sqrt{ }(2 \pi z) e^{z} K_{m}(z),
$$

it is found that

$$
\begin{aligned}
\int_{0}^{\infty} e^{-(1-z) \lambda} K_{n}(\lambda) K_{m}(z \lambda) \lambda^{k-1} d \lambda & \\
= & \frac{\pi 2^{-k} \sin k \pi z^{-\frac{1}{2}}}{\cos m \pi \cos (k+n) \pi \cos (k-n) \pi} E\left(\begin{array}{l}
1-k, \frac{1}{2}+m, \frac{1}{2}-m: e^{ \pm i \pi} z \\
\frac{3}{2}-k+n, \frac{3}{2}-k-n
\end{array}\right) \\
& -\sum_{n,-n} \frac{\pi 2^{-k-1} z^{-k-n}}{\sin n \pi \cos m \pi \cos (k+n) \pi} E\left(\begin{array}{l}
\frac{1}{2}+n, k+m+n, k-m+n: e^{ \pm i \pi} z \\
1+2 n, \frac{1}{2}+k+n
\end{array}\right)
\end{aligned}
$$

where $R(k \pm m \pm n)>0$.

\section{REFERENCES}

(1) MacRobert, T. M., Proc. Glasg. Math. Ass., 1 (1953), p. 187.

(2), (3) MacRobert, T. M., Proc. Glasg. Math. Ass., 1 (1953), p. 191.

(4) Ragab, F. M., Proc. Glasg. Math. Ass., 2 (1954), p. 85.

\section{UnTVERSity of GLasgow}

